\title{
Additive pulse mode-locking using a birefringent optical fiber
}

\author{
Sandra F. Feldman ${ }^{1}$, B.A. Moore, Doreen A. Weinberger ${ }^{2}$ and Herbert G. Winful \\ Department of Electrical Engineering and Computer Science, University of Michigan, Ann Arbor, MI 48109, USA
}

Received 4 May 1993; revised manuscript received 26 August 1993

\begin{abstract}
A powerful and increasingly common method to mode-lock a variety of lasers is additive pulse mode-locking (APM). In this technique, a nonlinear medium is placed in an external cavity which is coupled to the main laser cavity. As a result of interference at the common mirror between the pulses returning from each cavity, significant pulse shortening may be obtained. Optical fiber is often used as the nonlinear medium, and polarization effects and fiber birefringence are generally neglected. A computer model has been developed in order to simulate the effect of using a birefringent fiber. The simulations indicate that although the birefringence often has little qualitative effect on the system, in certain cases the birefringence may be exploited for enhanced modelocking.
\end{abstract}

\section{Introduction}

Additive pulse mode-locking (APM), also known as coupled cavity mode-locking, is an all-optical, interferometric method which may produce shorter pulses at higher repetition rates than other, more established, mode-locking techniques. A nonlinear external cavity is coupled to the main laser cavity. Pulse narrowing occurs as pulses in the laser cavity and the external cavity coherently interfere at the common mirror [1]. Generally, the external cavity functions as a nonlinear mirror which becomes highly reflective for intense light but which is less reflective for weak signals. Intense pulses thus receive preferential treatment compared to any other radiation in the laser cavity. The technique is very versatile since any nonlinear medium may be used in the external cavity. Optical fiber is used most frequently because it is relatively inexpensive, easy to use, and supplies sufficient nonlinearity across a broad band of wavelengths. Furthermore, optical fiber is very effective because it imparts an intensity dependent phase to the pulse in the external cavity so that the pulses in-

1 Currently at General Electric Corporate Research and Development Laboratories, Schenectady, NY 12309, USA.

2 Currently at Department of Physics, Smith College, Northamption, MA 01063, USA. terfere constructively at their peaks and destructively in the wings. Since in many laser systems APM is self starting, no active modulation need be applied in the main laser cavity to initiate the process [2]. An additional advantage of APM in some systems is that the femtosecond pulses produced appear to be highly chirped and may immediately be compressed further by using dispersion compensation [3]. Alternately, chirp compensating elements may be put directly into the external cavity to further reduce the pulse width [4]. The most significant drawback to APM, as in other interferometric techniques, is that a specific length relationship between the laser and the external cavity must be precisely maintained. A simple electronic servo loop must be used to maintain stable mode-locking for an extended period of time [5].

\section{Motivation for including a birefringent fiber in the external laser cavity}

The role of birefringence has been neglected in nearly all of the theoretical and experimental research on APM in which a fiber supplies the external cavity nonlinearity. The propagation of a light pulse in a birefringent fiber may be very complicated and the output polarization state of the light depends 
strongly on the fiber birefringence. An analysis of the effects of birefringence in an APM system is necessary to investigate both beneficial and detrimental effects birefringence may have on the final pulse width and the system stability However, in the theory typically a scalar approach is used which assumes linearly polarized light in the system, while in experiments one of two approaches is taken. In the first approach an isotropic fiber is used and the role of the light polarization is simply ignored. In the second approach, a highly bire fringent, polarization preserving fiber is used and the light is aligned along one of the fiber principal axes in order to maintain strictly linear polarization th roughout the experiment.

Most lasers are polarization sensitive due to the placement of elements at Brewster's angle within the cavity, In a simple implementation of APM with a birefringent fiber, one may try to exploit the nonlinear birefringence in order to obtain enhanced pulse shortening. Due to intensity dependent, light induced changes in the fiber refractive indices and hence in the fiber birefringence, the more intense central region of the pulse returns with a different polarization than the less intense wings [6]; it should be possible to use the polarization sensitive elements to chop the wings off the pulse in the main laser cavity without significantly affecting the pulse peak. Another potential source of pulse shaping arises due to the effects of cross-phase modulation between the two orthogonally polarized components of the pulse. Generally, an intensity dependent phase shift is induced across the pulse due to self-phase modulation by the nonlinear refractive index. More complicated and perhaps more effective phase modulation could be induced because of the interaction between the cross- and self-phase modulation. A properly oriented birefringent fiber might lead to more effective mode-locking than an isotropic fiber.

Unstable and perhaps chaotic dynamics may be expected if the fiber in the external cavity is oriented so as to probe the fast axis polarization instability [7]. One might expect the pulse to narrow smoothly, simultaneously increasing in peak power, until the pulse is intense enough to excite the polarization instability. Once the instability was excited, complicated dynamics would be expected until the pulse peak was below the instability threshold. The whole sequence might be expected to repeat indefinitely.

In order to explore these effects, we devised a computational model to investigate the dynamics of an APM laser with a nonlinear birefringent fiber in the external cavity. In general, the birefringence had less of an effect than anticipated. In many cases, the birefringence did not affect the qualitative dynamics significantly; the final pulse widths after 2000 round trips through the system were nearly identical to the pulse widths that were obtained using an isotropic fiber in the external cavity. However, in certain select cases the birefringence had a strong, significant effect. Output pulses were stabilized by increasing the birefringence and orienting the linearly polarized light exiting the laser cavity in an angular range between approximately $30^{\circ}$ and $60^{\circ}$ to the principal fiber axes. Decreasing the birefringence or aligning the light closer to the axes tended to cause the output to be highly unstable with periodic pulse spikes or to be stable but with large satellite pulses riding on the wings of the main pulse. The effect of the fast-axis polarization instability on the system dynamics was not satisfactorily probed, since it proved to be nearly impossible to obtain stable pulses of sufficient intensity to excite the instability. Although reducing the fiber birefringence is an alternative to increasing the pulse intensity, reducing the birefringence requires a long fiber in order to maintain one beat length of fiber in the cavity. An increase in fiber length leads to increased nonlinearity in the external cavity, and even along the axes stable, mode-locked pulses could not be maintained. In many cases the fiber birefringence has little effect on APM and the assumptions made in much of the theoretical and experimental work are valid. However, in certain cases the presence of birefringence enhances mode-locking. Adjusting the input orientation of the light to a birefringent fiber as well as the interferometric phase mismatch between the cavities may lead to improved mode-locked pulses.

\section{Theoretical model}

\subsection{Homogeneous laser model}

Blow and Wood were among the first to successfully model the pulse narrowing obtained by cou- 
pling a nonlinear external cavity to a laser and to extract the important dynamics of that system [8]. Based on their results which showed that the exact laser model does not affect the qualitative behavior of an APM laser, the same, simplified homogeneous laser model that they describe is used in this work. The model includes effects due to saturable gain, bandwidth considerations (also known as gain dispersion ), synchronously pumped mode-locking, and destabilizing, additive noise. After one round trip through the gain medium each of the polarization components $E_{x}$ and $E_{y}$ of the laser field is described in frequency space by

$\tilde{E}^{(n+1)}=\frac{\alpha \tilde{E}^{(n)}}{\left(1+I / I_{\text {sat }}\right)\left(1+\beta^{2} \omega^{2}\right)}+\Delta \frac{\mathrm{d}^{2} \tilde{E}^{(n)}}{\mathrm{d} \omega^{2}}+N$,

where $\alpha$ is the small signal gain, the total intensity $I=\int\left(\left|E_{x}\right|^{2}+\left|E_{y}\right|^{2}\right) \mathrm{d} t$ and $I_{\text {sat }}$ is the saturation intensity which controls the average laser output power. Typically the small signal gain $\alpha=2$, and the saturation intensity is in the range $50 \leqslant I_{\text {sat }} \leqslant 100$. The parameter $\beta$ is inversely related to the effective laser bandwidth due to the gain bandwidth and any intracavity filters. The additive noise $N$ is generally represented as random, white phase noise of the form

$N(\omega)=N_{0} \exp [i 2 \pi R(\omega)]$,

where $N_{0}$ is the constant noise amplitude and $R(\omega)$ varies randomly between 0 and 1 . This random phase noise serves as the seed to start the laser and also helps to establish the stability of the mode-locked pulses against a weak noise source. In this sense, the noise serves as a computational substitute for spontaneous emission in the laser. $\Delta$ represents the relative strength of synchronously pumped mode-locking. Keeping $\Delta$ small but nonzero helps to lessen the number of round trips required to reach steady state when the APM laser model is being started from noise. Additionally, a nonzero $\Delta$ helps to keep the pulse near the center of the computational time domain. When $\Delta=0$ the pulse is free to wander in time. Choosing $\beta=0.02$ and $\Delta=0.002$ yields a final steady state pulse width of about 1.1. Equation (1) is best evaluated in a split-step manner. First the effects of the saturable gain and noise (the first and third terms on the right hand side) may be applied in the frequency domain exactly as written in eq. (1). Then the effect of the synchronous mode-locking should be applied in the time domain.

Because elements in the laser cavity such as the gain crystal and the birefringence tuning plates are oriented at Brewster's angle, the effect of preferential transmission of one polarization component over the other must be considered. The notation is chosen such that the elements are positioned so as to fully transmit $x$-polarized light and to partially transmit $y$-polarized light. Assuming that the cavity elements are similar to glass and have indices of refraction of about 1.5 and treating them as thin plates (which is an excellent approximation for all but a tunable etalon), the fraction of the intensity of the $y$-polarized light transmitted through both Brewster surfaces of a given element is approximately 0.73 [9]. The corresponding field amplitude is reduced by a factor of 0.85 . Using this definition for the axes and referring to fig. 1, it is apparent that $E_{x, \text { las }}^{\prime}$ traverses the laser cavity essentially unchanged except for the effects of the gain medium. Meanwhile, $E_{y, \text { las }}^{\prime}$ is strongly attenuated. In a typical system, $E_{y, \text { las }}^{\prime}$ may first pass completely through three thin elements positioned at Brewster's angle, into the gain medium which is a crystal positioned at Brewster's angle, through the gain medium, out of and then back into the crystal (again through Brewster's angle), and, finally, on its exit from the crystal back to the output coupler, through another 3.5 Brewster angled elements (one half of the gain crystal and three thin elements). Since

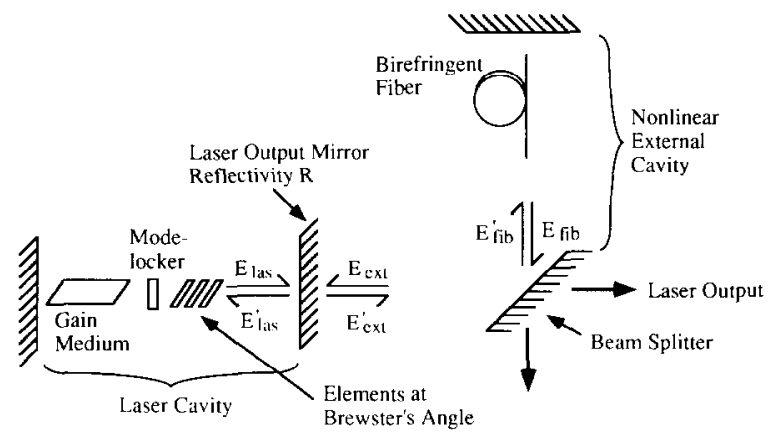

Fig. 1. Schematic of an APM laser which illustrates the notation and main components of the model. The laser output is taken through the beamsplitter as shown. A length of birefringent optical fiber is used to supply the nonlinearity in the external cavity, while the main laser cavity contains the gain medium and mode-locker, as well as windows and tuning elements oriented at Brewster's angle which are shown simply as angled flats. 
the aim of this paper is to pror ide a simplified functional model and to extract the qualitative aspects of APM with a birefringent fiber, a slightly simplified path for the $y$-polarized component is used. Beginning at the common mirror setween the cavities, $E_{y, \text { las }}^{\prime}$ passes through four elements positioned at Brewster's angle and is reduced to $(0.85)^{4} E_{y \text {, las }}^{\prime} \approx$ $0.52 E_{y, \text { las }}^{\prime}$. Then $E_{y, \text { las }}^{\prime}$ makes one round trip through the gain medium. Finally $E_{y, \text { las }}^{\prime}$ passes again through the four elements at Brewster's angle on its return to the output coupler. With this implification, the total field entering the gain medium is $E_{\text {las }}^{\prime}=$ $E_{x, \text { las }}^{\prime} \hat{x}+0.52 E_{y, \text { las }}^{\prime} \hat{y}$. After mi,king the round trip through the gain medium by a pplying eq. (1) to the fields, the effect of the Brews er angled elements is again calculated. This field is mixed with the field returning from the external cavity. The overall effect of these many elements at Breivster's angle is to very nearly suppress any lasing in the $y$-polarization. The alignment of the fiber to the lisser will be defined as the fiber orientation relative to this preferred $x$ polarization.

The effect of birefringent elements in the laser cavity on the polarization of the pulse has been completely neglected. The effect $\mathrm{cf}$ cavity birefringence is expected to be small compa ed to the effect of the polarization selectivity of the Brewster angled elements. Cavity birefringence wculd lead to a net phase difference between the two polarization components. Since the laser tends tc lase primarily in the $x$ polarization a net phase difference between the $x$ and $y$ polarizations is of little consequence.

\subsection{Pulse propagation in optical fiber}

In normalized units and in $x$ frame moving at the average group velocity $\bar{v}_{\mathrm{g}}$ of the polarization components $u$ and $v$, the coupled :onlinear Schrödinger equation for pulse propagatior in a birefringent fiber is [10]

$$
\begin{aligned}
& \mathrm{i}\left(\frac{\partial u}{\partial \xi}+\delta \frac{\partial u}{\partial s}\right) \pm \frac{1}{2} \frac{\partial^{2} u}{\partial s^{2}}+\left(|u|^{2}+\frac{2}{3}|v|^{2}\right) u \\
& \quad+\frac{1}{3} v^{2} u^{*} \exp (-\mathrm{i} R \delta \xi)=0, \\
& \mathrm{i}\left(\frac{\partial v}{\partial \xi}-\delta \frac{\partial v}{\partial s}\right) \pm \frac{1}{2} \frac{\partial^{2} v}{\partial s^{2}}+\left(\frac{2}{3}|u|^{2}+|v|^{2}\right) v \\
& \quad+\frac{1}{3} u^{2} v^{*} \exp (\mathrm{i} R \delta \xi)=0,
\end{aligned}
$$

where $u=\left(\chi z_{0} / \pi\right)^{1 / 2} U$ and $v=\left(\chi z_{0} / \pi\right)^{1 / 2} V$ are the electric field components along the slow and fast fiber axes, the normalized time $s=\left(t-z / \bar{v}_{\mathrm{g}}\right) / t_{0}$, the normalized distance in the direction of propagation $\xi=\pi z / 2 z_{0}$, the birefringence $\delta=\pi c \Delta n t_{0} / D(\lambda) \lambda_{0}$, and $R=8 \pi c t_{0} / \lambda_{0}$ where $t_{0}=0.568 \tau$. The soliton period $z_{0}=\pi^{2} c^{2} t^{2} / D(\lambda) \lambda_{0}$. The first pair of terms in eqs. (3) allows for propagation in $\xi$, the second term leads to dispersion, the third term represents self- and crossphase modulation due to the nonlinear refractive index, and the final term accounts for nonlinear ellipse rotation. The $+/-$ sign in the dispersive term corresponds to normal/anomalous dispersion. Numerical parameters for these equations are typically assigned as follows. The parameter $\tau$ is chosen $\tau=5 \mathrm{ps}$ so that a pulse defined as $U=\operatorname{sech}(s)$ would have a fwhm pulse width of $5 \mathrm{ps}$. At the wavelength $\lambda_{0}=1.55$ $\mu \mathrm{m}$ the dispersion $D(\lambda)=6.5 \times 10^{-3}$ which corresponds to $-20 \mathrm{ps}^{2} / \mathrm{km}$. The Kerr coefficient $\chi=$ $2 n_{2} \omega_{0} / c A_{\text {eff }}$ includes a geometric factor due to the fiber core size and is about $1.32 \times 10^{-2} \mathrm{~m}^{-1} \mathrm{~W}^{-1}$. Using these values, the normalized pulse intensity is related to the physical pulse intensity by $U=$ $0.6 u \sqrt{\mathrm{W}}$. (The intensity $|U|^{2}=0.36|u|^{2} \mathrm{~W}$.) The fiber birefringence $\Delta n$ is varied to include very weakly birefringent fibers $\left(\delta=0.008, \Delta n \approx 3 \times 10^{-8}\right)$ and highly polarization preserving fiber $(\delta=0.8$, $\Delta n \approx 3 \times 10^{-6}$ ) as well as isotropic fiber. The fiber length is chosen to be about the same as the main laser cavity length. Additional nonlinearity may be obtained by using a longer fiber, but then more than one pulse will be propagating in the external cavity at one time. A rather long fiber length of $3.6 \mathrm{~m}$ is used, but only one pass is made through the fiber rather than the usual round trip. The effect of the retroreflector which normally terminates the fiber in an APM laser is thus neglected.

By transforming to a circularly polarized basis the exponential from the final terms in eqs. (3) is eliminated $^{\# 1}[11]$. The resulting equations may be numerically investigated using a split-step Fourier technique. The propagation terms are implemented in frequency space, while the nonlinearity is applied in

\# The method used to eliminate the exponential term is identical to the method outlined by Blow, Doran and Wood [11]. Note, however, that the equations here are slightly different than those used by Blow et al. 
the time regime at the midpoint of each propagation step. The simulations are checked for accuracy by doubling the number of points used in the time mesh and by halving the step size used in the propagation steps. In addition, the Fortran code has been checked for accuracy by investigating various limiting cases and by comparison with published literature.

\subsection{Coupling between the laser and external cavities}

The fields on either side of the common mirror between the external and the laser cavities are related by the following set of equations

$$
\begin{aligned}
& \boldsymbol{E}_{\text {las }}^{\prime}=r \boldsymbol{E}_{\mathrm{las}}+\sqrt{1-r^{2}} \exp (\mathrm{i} P \pi) \boldsymbol{E}_{\mathrm{ext}}, \\
& \boldsymbol{E}_{\mathrm{ext}}^{\prime}=\sqrt{1-r^{2}} \boldsymbol{E}_{\mathrm{las}}-r \exp (\mathrm{i} P \pi) \boldsymbol{E}_{\mathrm{ext}} .
\end{aligned}
$$

The notation is clarified by the laser schematic shown in fig. 1. Primed fields are outgoing and unprimed fields are incoming relative to the common mirror. Neither fiber coupling nor output coupler losses have been explicitly written as part of eqs. (4). The parameter $r$ is the field reflectivity of the common mirror, while $P$ is the cavity length mismatch as discussed further in the next paragraph. The field reflectivity $r$ is generally set to 0.7 for an intensity coupling of 0.5 . Low values for the reflectivity lead to stronger coupling between the cavities. Unlike in previous work, the fields $\boldsymbol{E}$ are vector quantities since they may have arbitrary polarizations. Equations (4) must be applied to the $x$ and $y$ components of each field $E_{\text {las }}$ and $E_{\text {ext }}$.

If the cavities are not precisely the same length, this will be manifested in a phase difference, referred to as the phase bias, between the pulses returning to the common mirror from the two cavities. The phase bias must be chosen carefully in order to obtain optimum pulse narrowing when the two circulating pulses are combined. For example, a phase bias of $-\pi / 2$ in a system with self-phase modulation (SPM) but no dispersion combines the pulses completely additively at their peaks if the fiber length and pulse power is adjusted so that the peak phase shift due to the SPM is $\pi / 2$. Then the addition is out of phase everywhere but the peak which leads to dramatic pulse narrowing [12]. The cavity mismatch is explicitly included as the parameter $P$ in eqs. (4). To get one full wavelength of interferometric variation between the cavities, $P$ varies such that $0 \leqslant P<2$. Thus, a phase mismatch of $2 \pi$ corresponds to a cavity length mismatch of exactly one wavelength, measured at the central operating wavelength of the laser system.

In order to complete the system, the fields entering and leaving the external cavity must be related to the fields entering and leaving the fiber. The main source of loss is the output coupler and coupling loss to the fiber. Lumping these together as $\theta$, assuming that they are reciprocal, and assuming that there is no polarization sensitivity in the output coupler

$E_{\text {fib }}^{\prime}=\mathrm{i} \theta E_{\text {ext }}^{\prime}$,

$\boldsymbol{E}_{\text {ext }}^{\prime}=\mathrm{i} \theta \boldsymbol{E}_{\mathrm{fib}}$.

Typical values for the output coupler would be 0.9 , and in the computation the fiber insertion loss is neglected.

\section{Results of the numerical simulations}

\subsection{General computation technique}

The laser start-up from the random phase noise is investigated in this work. Another option would have been to start the model with a pulse corresponding to the steady state operating point of the laser alone. This would correspond physically to turning on the laser and allowing it to reach steady state before unblocking the extcrnal nonlinear cavity. A final option would have been to try to start the model with a perturbed version of a known steady state operating point of the APM laser in order to investigate the stability of the system.

The three pieces of the APM model, namely the homogeneous laser model, the equations governing pulse propagation in a birefringent fiber, and the coupling between the laser and external cavities, have been described. To investigate lasing beginning from the random phase noise inherent in the laser model, initially all fields in the system are set to zero. The field components then make one round trip through the gain medium. The resulting noise impinges on the common mirror and is split between the two cavities. After the fields propagate through each cavity 
they recombine coherently at the common mirror with some phase difference due to the cavity length mismatch. The process continues in this way until about 2000 round trips have been simulated. This appears to be a sufficient number of round trips to determine whether a steady stc.te pulse has been (or will be) reached or whether the laser will oscillate only in noisy, erratic spikes. The effect of the cavity mismatch, the fiber birefringence, and other laser parameters may be thoroughly investigated.

In order to evaluate the effectiveness of the external cavity in inducing mode-locking, the pulse width must be measured. For convenience the pulse width is measured inside the laser cavity. In the early stages of pulse formation, or if the pulse never reaches steady state, the pulse is extremely ill-defined. Even when steady state is reached after many round trips through the APM laser, double-humped pulses, pulses with satellite pulses riding on their wings, or similarly odd shapes pulses sometimes result. When working with" such pulses, it is difficult to use the standard fwhm definition for the pulse width. Therefore, all pulse widths are presented in terms of the RMS (root mean square) pulse width which is defined as the total pulse intensity divided by the peak pulse intensity

$$
\begin{gathered}
\tau_{\mathrm{RMS}}=\left(\int_{-\infty}^{\infty}\left(\left|E_{x}\right|^{2}+\left|E_{y}\right|^{2}\right) \mathrm{d} t\right) \\
\times\left[\max \left(\left|E_{x}\right|^{2}+\left|E_{y}\right|^{2}\right)\right]^{-1} .
\end{gathered}
$$

In a smooth, near-gaussian pulse the fwhm pulse width is about 0.7 times the RMS pulse width.

\subsection{APM using an isotropic fiber}

Before investigating the effects of a birefringent fiber on additive pulse mode-locking, the model was tested using an isotropic fiber and all polarization effects were neglected. This allows identification of those dynamics which are directly related to the fiber birefringence and those which are independent of birefringence.

The growth of a pulse in the laser cavity without any coupling to an external cavity is shown in fig. 2 . The parameters have been chosen so that the RMS pulse width is about 1.1 in normalized units, cor- responding to a 5 ps pulse. Examination of the figure shows that lasing begins from initial noise spikes, and that within approximately 300 round trips much of the structure and winglike appendages on the pulse disappear as it evolves into a smooth pulse. The phase of the pulse (fig. 2b) evolves over a much longer period. After approximately 1000 round trips, the phase is nearly flat (although nonzero) across the entire pulse. The pulse width continues to decrease very slowly throughout this process (fig. $2 \mathrm{~d}$ ). The pulse spectrum is seen in fig. $2 \mathrm{c}$.

A range of laser dynamics results when the nonlinear external cavity is coupled to the laser. The laser may produce wide pulses, may produce noise and never stabilize into pulsed output, or may produce double-humped pulses, or output with substantial energy in the pulse wings. By adjusting the relative coupling strength between the cavities, the reflectivity of the output coupler, and the phase mismatch between the cavities, the APM laser may be successfully mode-locked. The shortest pulses obtained in this simulation had an RMS pulse width of 0.2 , and were thus narrowed by more than a factor of five compared to the pulses produced by the laser alone. However, many researchers have reported narrowing by much greater factors than 5 , and the possible reasons for this discrepancy between this work and the experimental results include the following. Since the pulse width is sensitive to the various experimental parameters, it is difficult to determine the optimum values to be used in the simulation. A different choice for various values in the parameter space may have yielded greater narrowing. Additionally, approximations have been made in both the laser and fiber models for the sake of simplicity. Small, higher order effects which have been neglected (since the primary interest here is in examining the qualitative effect of birefringence) could possibly be important in obtaining the very shortest pulses. The pulse and spectral evolution are shown in fig. 3. As expected, a broadened spectrum is seen along with the narrower pulse.

Close examination of the data used to generate fig. 3 reveals that the mode-locked pulse is not centered at time $s=0$ as expccted. Instead, the peak of the pulse has shifted over to time $s=0.25$. This shift is due to an accumulation of phase on the pulse. The phase across the pulse (fig. 3 ) no longer evolves to- 

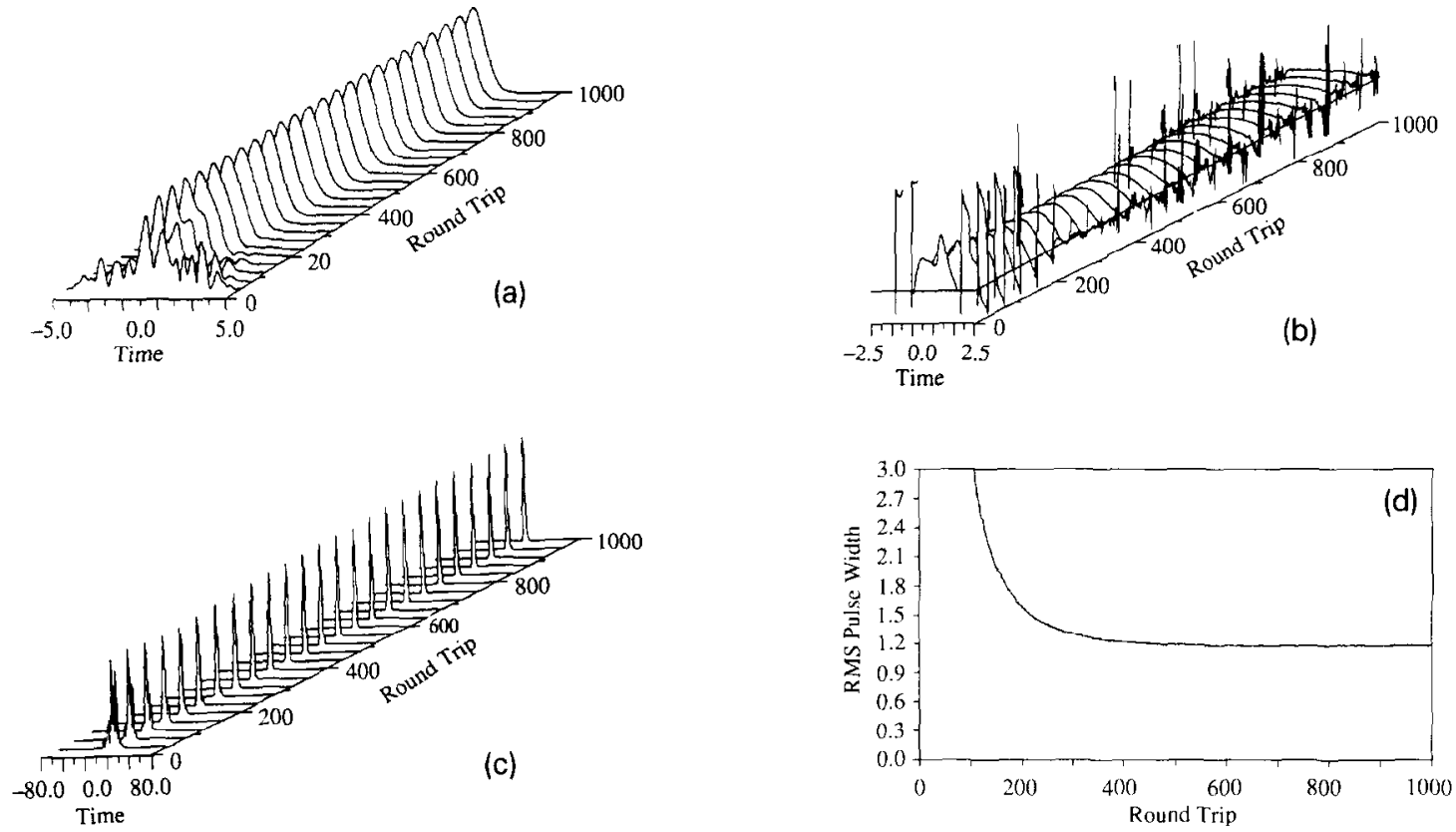

Fig. 2. Pulse amplitude evolution [a], phase evolution [b], spectrum [c], and RMS pulse width [d] for a synchronously pumped modelocked laser with no coupling to an external cavity. The small signal gain $\alpha=2.0$, the bandwidth parameter $\beta=0.02$, and the synchpumped mode-locking is weak with $\Delta=0.002$. The saturation intensity is chosen $I_{\text {sat }}=50$. Outside the pulse the phase is random owing to the effect of the additive noise used in the model. The phase develops a smooth profile across the pulse [b].

ward a constant as in the laser alone. Rather, the pulse is chirped, and accumulates a small amount of additional phase with each round trip. The observed time shift has not been reported in the literature. However, since the preferred method of detecting short pulses utilizes autocorrelation, a relative time shift of the pulse would not be readily apparent. The nearly linear chirp on the pulse is consistent with experimental research and would allow the APM laser pulses to be compressed further.

\subsection{APM using a birefringent fiber}

When a birefringent fiber rather than an isotropic fiber is used as the nonlinear medium in the external cavity, both the strength of the birefringence and the orientation of the principal fiber axes relative to the laser cavity will affect the pulse formation. Recall that the laser alone will produce linearly polarized output. In order to discuss the effect of fiber orientation, the angle between the slow axis of the birefringent fiber and this preferred laser polarization is referenced. Only two basic APM configurations need be examined in order to observe a range of birefringence related dynamics. At a low value of the saturation intensity $\left(I_{\text {sat }}=50\right)$ the birefringence appeared to have little effect. At higher laser power $\left(I_{\text {sat }}=100\right)$ the birefringence could be exploited to enhance the mode-locking of the system.

The less interesting dynamics were obtained using the same system as in fig. 3, but replacing the isotropic fiber with a birefringent fiber. During the course of the simulations, only the cavity phase mismatch, the fiber birefringence, and the orientation of the fiber were varied. No noteworthy effects were observed due to the birefringence. If the system achieved stable mode-locking with an isotropic fiber, it achieved stable mode-locking with the birefringent fiber. The number of round trips to achieve stable model-locking might vary, but stable output pulses would be achieved. Neither the pulse shape nor the pulse width was significantly affected. The position of the pulse peak in the time domain was dependent on both the birefringence and the fiber orientation. This occurs because changes in birefringence translate directly to changes in the effective cavity length. 

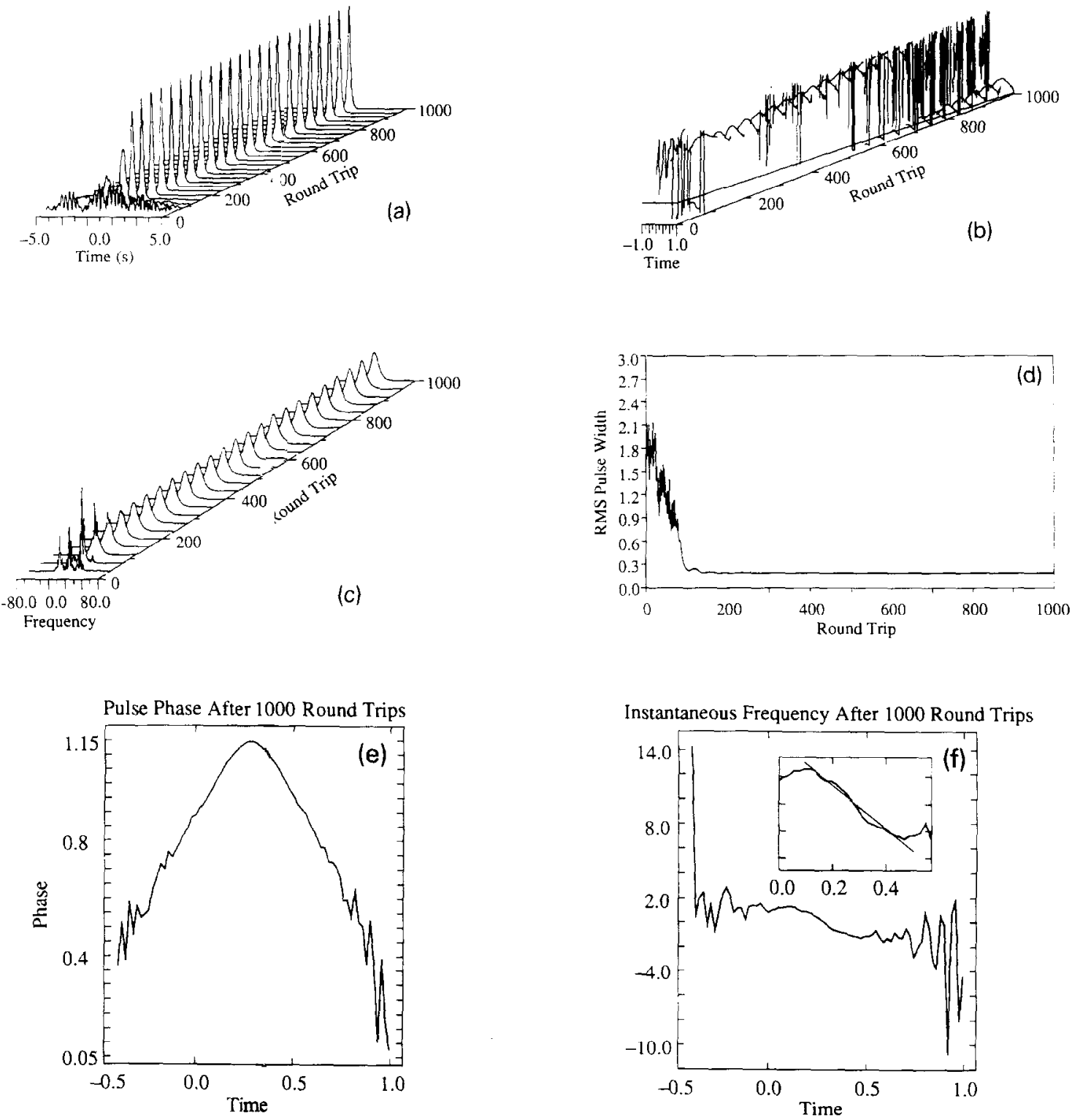

Fig. 3. Pulse amplitude evolution [a , phase evolution [b], spectrum [c], and RMS pulse width [d] for a synchronously pumped modelocked laser coupled to a nonlinear external cavity containing an isotropic fiber. The phase evolves to an approximately quadratic shape. Overall, the phase is a slowly increasing function. (After reaching $2 \pi$, the computation resets the phase to 0 .) The pulse phase [e] and instantaneous frequency [f] are shown for a pulse after 1000 round trips in the laser. The instantaneous frequency is the derivative with respect to time of the phase. The inset shows an expanded view of the pulse peak. A straight line has been superimposed to show that the chirp is nearly linear. As in fig. 2 the small signal gain $\alpha=2.0$, the bandwidth parameter $\beta=0.02$, and the synch-pumped mode-locking is weak with $\Delta=0.002$. The saturation ntensity is $I_{\text {sat }}=50$. The phase mismatch between the cavities $P=0.1$, the reflectivity of the common mirror $R=0.7$, and the output coupler strength $\theta=0.9$. 
A small change in length means a slightly different accumulation of phase on each round trip and hence a different position for the pulse in time. This shift is readily apparent for the two pulse evolutions for a highly birefringent fiber compared in fig. 4. In fig. 4a the laser output is aligned at $45^{\circ}$ to the fiber axes, while in fig. $4 \mathrm{~b}$ the output is aligned with the fiber fast axis. Because of the slightly lower average index of refraction along the fast axis, the effective control cavity length is reduced and the laser repetition rate is correspondingly slightly reduced. For relatively low powers of the laser intensity, the fiber birefringence and orientation had only insignificant quantitative effects on the exact nature of the pulse evolution rather than any substantial qualitative effect.

A more interesting configuration was obtained in an attempt to generate more intense pulses by raising
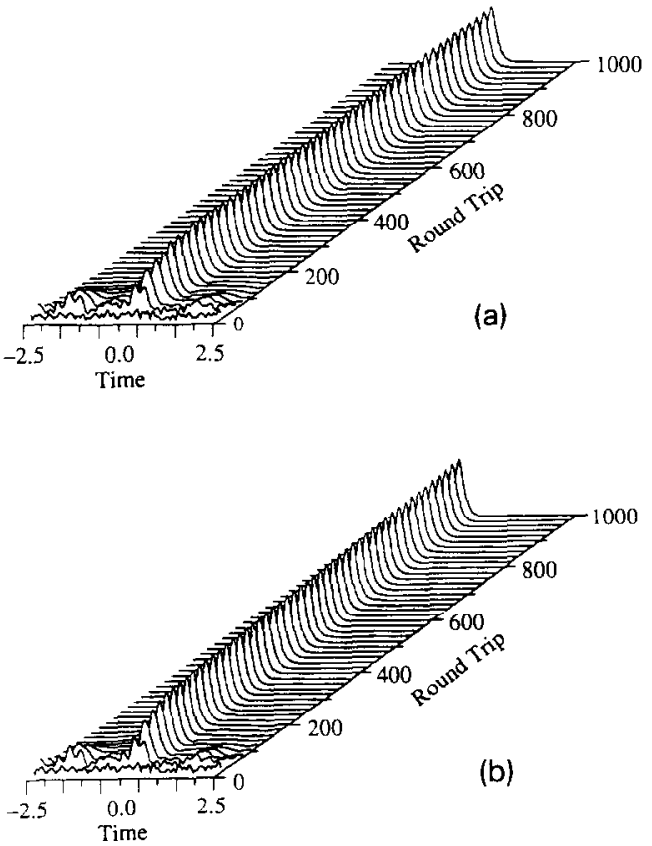

Fig. 4. The shift in pulse position obtained with an APM laser operating with a highly birefringent fiber. Pulse evolution when the fiber axes are aligned at $45^{\circ}$ to the laser [a ]. Pulse evolution when the fiber fast axis is aligned with the laser [b]. The pulse peak is clearly seen to slowly move toward a different position in time in each of the figures. The birefringence $\delta=0.5$, fiber length $\xi=0.008$, fiber step size $\Delta \xi=0.001$, phase mismatch $P=0.15$ and number of points in the time mesh $N=512$. The laser parameters are the same as in fig. 2. the saturation intensity of the laser. When the saturation intensity was doubled to $I_{\text {sat }}=100$, clean, narrow pulses were unattainable at any value of the cavity mismatch for an isotropic fiber in the external cavity. The best pulses obtained had a strong central pulse with a weak but significant satellite pulse riding on one wing. Despite this inability to generate clean pulses using an isotropic fiber, an investigation was undertaken to determine whether the birefringence might be exploited to achieve enhanced modelocking. The pulse shape improved and better modelocking was achieved by increasing the fiber birefringence and by positioning the fiber so that the approximately linearly polarized laser output was oriented between approximately $30^{\circ}$ and $60^{\circ}$ to the fiber principal axes. The most likely explanation for the enhanced mode-locking at higher power lies in the cross-phase modulation between the two polarization components propagating in a birefringent fiber; the effect is most pronounced when the relative intensities of the two components are about equal and when the fiber is strongly birefringent. Figures $5(\mathrm{a})-$ (f) compare the pulse evolutions for three different values of the fiber birefringence and two values for the fiber orientation for a phase mismatch which does not lead to mode-locking using the isotropic fiber. For each data set the fiber birefringence was increased by a factor of ten. At each value of the birefringence, the laser output was aligned at $10^{\circ}$ or at $40^{\circ}$ to the fiber slow axis. All other values for the system remain constant through the entire comparison. Figures $5(\mathrm{~g})$ and $5(\mathrm{~h})$ show the pulse evolution for a moderate level of birefringence, with the fiber orientation again at either $10^{\circ}$ or $40^{\circ}$, but for a different value of the phase mismatch. In this case, near the fiber axis (or for a weakly birefringent or isotropic fiber) mode-locked pulses may be obtained. However, significant energy is contained in a satellite pulse riding on the wings of the main modelocked pulse. When the fiber orientation is changed so that the laser output is nearly at $45^{\circ}$ to the fiber axis, clean, high-quality pulses are obtained. These findings may have important implications for experimenters working with APM lasers. Previously, if stable mode-locking proved elusive the primary adjustment which could be made was the interferometric phase mismatch between the two cavities. This research suggests that it may be helpful to use a bi- 

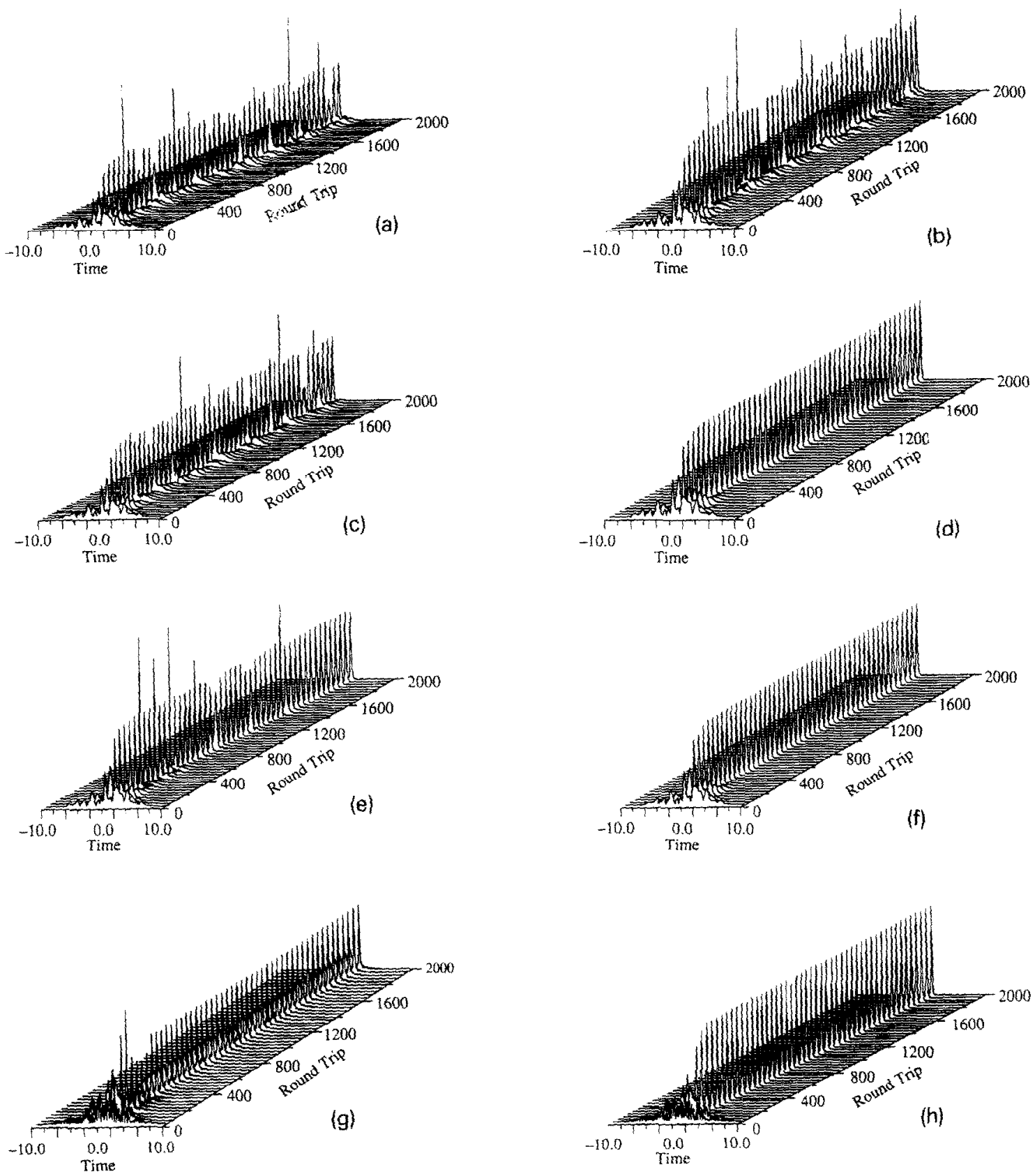

Fig. 5. Comparison of the pulse evolution for an APM laser with a birefringent fiber at a high laser intensity. Laser and fiber parameters are as described in the text. The saturation intensity $I_{\text {sat }}=100$. The phase mismatch between the cavities $P=0.3[\mathrm{a}-\mathrm{f}]$ and $P=0.1[\mathrm{~g} \sim \mathrm{h}]$. The fiber birefringence $\theta$ and angle between the slow axis and the laser are as follows: $\delta=0.008, \theta=10^{\circ}[\mathrm{a}], \alpha=0.008, \theta=40^{\circ}[\mathrm{b}]$, $\delta=0.08, \theta=10^{\circ}[\mathrm{c}], \delta=0.08, \theta=40^{\circ}[\mathrm{d}], \delta=0.8, \theta=10^{\circ}[\mathrm{e}], \delta=0.8, \theta=40^{\circ}[\mathrm{f}], \delta=0.08, \theta=10^{\circ}[\mathrm{g}], \delta=0.08, \theta \approx 40^{\circ}$ [h]. The fiber length is still $\xi=0.008$, but the step size has been decreased to $\Delta \xi=0.0001$ and the number of points increased to 1024. The mode-locking of the laser is clearly enhanced by greater birefringence and by aligning the fiber so that the polarized laser output is oriented close to $45^{\circ}$ from the fiber axes. Note that stable, high quality mode-locked pulses were unattainable using an isotropic fiber in the external cavity. 
refringent fiber and to adjust the orientation of its axes in order to achieve better, more stable modelocking and cleaner pulse shapes.

\section{Summary}

Since its first demonstration in 1984, additive pulse mode-locking (APM) has come into widespread use as a versatile, effective technique for generating ultrashort pulses in a variety of laser systems. The basic concept of APM is simple. One needs only to couple a nonlinear control cavity to the laser cavity and to adjust the interferometric length mismatch between the two cavities in order to achieve short pulses, since the coherent interference between the pulses at the common mirror between the cavities leads directly to the pulse narrowing. In practice, it is necessary to use active length stabilization to maintain the correct phase relationship between the two cavities.

A number of nonlinear media may be used in the external cavity, but most often optical fiber is used. To date, both the theory and experimental research presented has neglected the role played by the fiber birefringence. We have devised a model and developed a computer simulation in order to more fully investigate the effect of birefringence.

In the simulations the interrelated roles of the phase mismatch between the laser and external cavities, the strength of the birefringence, the orientation of the fiber axes relative to the preferred polarization of the laser output, and the encrgy in the pulse were investigated. Other adjustable parameters such as the reflectivity of the common mirror between the cavities, the reflectivity of the output coupler, and the fiber length were held constant. In many cases, particularly when the pulse energy was low, the birefringence had little effect on the APM system. Quantitative details such as the number of round trips to achieve steady state mode-locking or the exact position of the pulse in time would change as a consequence of the birefringence. However, if the system could be stably mode-locked using an isotropic fiber, it could be stably mode-locked, without significant difference or improvement using a birefringent fiber. Changing the birefringence or the orientation of the fiber axes was often simply equiva- lent to a small change in the effective length of the fiber. However, when the pulse energy was increased in an attempt to increase the nonlinear effects in the fiber, the birefringence could be used to enhance the mode-locking. At the increased saturation intensity, the isotropic fiber used alone was unable to satisfactorily mode-lock the laser. Depending on the choice of the cavity phase mismatch, the output varied. Often, only unstable laser spiking was obtained. The best pulses were narrower than those produced by the laser alone, but had a large secondary pulse riding on the wings of the main pulse. When a fiber with substantial birefringence was place in the external cavity and the fiber axes were oriented in the general vicinity of $45^{\circ}$ to the polarized laser output, stable, short, mode-locked pulses were immediately obtained. If either of the fiber axes were aligned at only a few degrees to the laser output, or if the fiber was not birefringent enough, high quality, modelocked output pulses were not obtained.

To a large degree, the APM community's neglect of the role of fiber birefringence has been justified. In many systems, it appears that the birefringence has no significant effect. According to our simulations, however, the fiber birefringence may be exploited to enhance the mode-locking in particular systems. It is not unreasonable to suggest that researchers using an APM laser consider the fiber birefringence and orientation to be an additional, adjustable parameter of their system.

\section{Acknowledgement}

This work is based on research supported under a National Science Foundation Graduate Fellowship to Sandra F. Feldman. The authors acknowledge National Science Foundation support under grants EET8552520 (Doreen A. Weinberger) and EET-8712877 (Herbert G. Winful).

\section{References}

[1 ] J. Mark, L.Y. Liu, K.L. Hall, H.A. Haus and E.P. Ippen, Optics Lett. 14 (1989) 48.

[2] E.P. Ippen, L.Y. Liu and H.A. Haus, Optics Lett. 15 (1990) 183. 
[3] J. Goodberlet, J. Wang, J.G. Fujimoto and P.A. Schulz, Optics Lett. 14 (1989) 1125.

[4] X. Zhu, A. Finch and W. Sibbett, J. Opt. Soc. Am. B 7 (1990) 187.

[5] F.M. Mitschke and L.F. Mollenauer, IEEE J. Quantum Electron. QE-22 (1986) 2242.

[6] R.H. Stolen, J. Botineau and A. Ashkin, Optics Lett. 7 (1982) 512.

[7] S.F. Feldman, D.A. Weinberger and H.G. Winful, Optics Lett. 15 (1990) 311.
[8] K.J. Blow and D. Wood, J. Opt. Soc. Am. B 5 (1988) 629.

[9] M. Born and E. Wolf, Principles of optics: electromagentic theory of propagation, interference, and diffraction of light (Pergamon Press, New York, 1983).

[10] C.R. Menyuk, J. Opt. Soc. Am. B 5 (1988) 392.

[11] K.J. Blow, N.J. Doran and D. Wood, Optics Lett. 12 (1987) 202.

[12] E.P. Ippen, H.A. Haus and L.Y. Liu, J. Opt. Soc. Am. B 6 (1989) 1736. 